Kong. Res. J. 3(2): 1-6, 2016

ISSN 2349-2694

Kongunadu Arts and Science College, Coimbatore.

\title{
NONLOCAL CONTROLLABILITY OF IMPULSIVE FUNCTIONAL INTEGRO-DIFFERENTIAL EQUATIONS IN BANACH SPACES
}

\author{
Ravichandran C. ${ }^{1}$ and N. Valliammal ${ }^{2}$ \\ ${ }^{1}$ Department of Mathematics, KPR Institute of Engineering and Technology, Coimbatore - 641407. \\ ${ }^{2}$ Department of Mathematics, Sri Eshwar College of Engineering,Coimbatore- 641202. \\ E.mail: ravibirthday@gmail.com; vallikrishva@gmail.com
}

\section{ABSTRACT}

The paper is concerned with the controllability of impulsive functional integrodifferential equations with nonlocal conditions. Using the measure of noncompactness and Monch fixed point theorem, we establish some sufficient conditions for controllability and also our theorems extend some analogous results of (impulsive) control systems.

Keywords: Noncompactness, Integrodifferential equations.

\section{INTRODUCTION}

Impulsive differential equations are a class of important models which describes many evolution process that abruptly change their state at a certain moment,see the monographs of Bainov and Simonov (1993), Lakshmikantham et al. (1989) and have been studied extensively by many authors (Cuevas et al., 2009; Fan and Li, 2010; Anguraj and Mallika Arjunan, 2009). On the other hand, the concept of controllability is of great importance in mathematical control theory. Many authors have been studied the control of nonlinear systems with and without impulses; see for instance (Guo et al., 2004; Chen and Li, 2010; Ji et al., 2011).

The starting point of this paper is the work in papers (Ji et al., 2011; Jose et al., 2013). Especially, authors in Jose et al. (2013) investigated the controllability results of mixed-type functional integro-differential evolution equations with nonlocal conditions

$$
\begin{aligned}
& \quad x^{\prime} t \\
& =A t x t \\
& +f t, x_{t}, \quad t, s, x_{s} d s, k t, s, x_{s} d s \\
& \quad+B u t, \\
& t \in J=0, b, t \neq t_{i}, i=1, \ldots, s, \\
& \left.\quad \Delta x\right|_{t=t_{i}}={ }_{i} x_{t_{i}}, i \\
& \quad=1, \ldots, s, \\
& x_{0} \\
& =\varnothing+g x, t \epsilon-r, 0,
\end{aligned}
$$

by using Monch fixed point theorem. And in (Ji et al., 2011), authors studied the following controllability of impulsive differential systems with nonlocal conditions of the form

$$
\begin{aligned}
& x^{\prime} t=A t x t+f t, x t+B u t \text { a.e } \\
& 0, b \\
& \quad \Delta x t_{i}=x t^{+}{ }_{i} x t^{-}=I_{i i} x t_{i}, i \\
& =1, \ldots, s . \\
& x 0+M x \\
& \quad=x_{0}
\end{aligned}
$$

Motivated by above mentioned works (Ji et al., 2011; Jose et al., 2013), the main work of this paper is to prove the controllability results of impulsive integrodifferential systems with nonlocal conditions.

$$
\begin{aligned}
& x^{\prime} t=A t x t+f t, x t \\
& +{ }_{t} t, s, x(s) d s \\
& +R^{2} t \\
& \Delta x t_{i}=x t^{+} \rightarrow x t^{-}=I_{i i} x t_{i}, i \\
& =1, \ldots, s . \\
& x 0+M x \\
& =x_{0}
\end{aligned}
$$

Where $A t$ is a family of linear operators which generates an evolution operator

$$
\begin{gathered}
U t, s: \Delta=t, s \in 0, b \times 0, b: 0 \leq s \leq t \leq b \\
\rightarrow L X,
\end{gathered}
$$

here, $\mathrm{X}$ is a banach space, $L X$ is the space of all bounded linear operators in $X ; f: 0, b \times X \rightarrow$ $X ; G: 0, b \times X \rightarrow X ; O<t_{1}<\ldots \ldots . .<t_{s}<t_{s+1}=$ $b ; I_{i}=X \rightarrow X, i=1, \ldots$, sare impulsive functions; $M: P C \quad 0, b ; X \rightarrow X ; \quad \mathrm{B} \quad$ is a bounded linear 
operators from a Banach space $V$ to $X$ and the control function $u(\cdot)$ is given in $L^{2}(0, b, V)$.

The paper is organized as follows: In section 2 , we will recall some basic notations definition, hypothesis and necessary preliminaries. In section 3, we prove the controllability of impulsive integrodifferential system with nonlocal system(1.7) -(1.9), usingMonch fixed point theorem.

\section{PRELIMINARIES}

In this section, we recall some basic definitions and lemmas which will be used to prove our main results of this paper.

Let $(X,$.$) be a real banach space.We$ denote by $\mathrm{C}([0, \mathrm{~b}] ; \mathrm{X})$ the space of $\mathrm{X}$ - valued continuous function on $[0, \mathrm{~b}]$ with the norm $x=$ $\sup \{x(t), \mathrm{t} \varepsilon[0, \mathrm{~b}]\}$ and by $L^{1}([0, \mathrm{~b}] ; \mathrm{X})$ the space of $\mathrm{X}$ - valued Bochnerintegrable functions on $[0, \mathrm{~b}]$ with the norm $f_{1}=_{L}^{b} f\left(t_{0}\right) \mathrm{dtS}$.

For the sake of simplicity, we put $\mathrm{J}=[0, \mathrm{~b}]$; $J_{0}=\left[0, t_{1}\right] ; J_{i}=\left(t_{i}, t_{i+1}\right], \mathrm{i}=1, \ldots \ldots, \mathrm{s}$. In order to define the mild solution of problem (1.7)-(1.9), we introduce the set $\mathrm{PC}([0, \mathrm{~b}] ; \mathrm{X})=\{\mathrm{u}:[0, \mathrm{~b}] \rightarrow \mathrm{X}: \mathrm{u}$ is $]$; continuous on $J_{i}, \mathrm{i}=0,1, \ldots, \mathrm{s}$ and the right limit $\mathrm{u}\left(t_{i}^{+}\right)$ exists, $\mathrm{i}=1 \ldots ., \mathrm{s}\}$. It is easy to verify that $\mathrm{PC}([0, \mathrm{~b}] ; \mathrm{X})$ is a banach space with the norm $u_{P C}=\sup \{u(t), t$ $\varepsilon[0, \mathrm{~b}]\}$.

Definition 2.1: Let $E^{+}$be the positive cone of an order Banach space $(E, \leq)$. A function $\Phi$ defined on the set of all bounded subsets of the Banach space $X$ with values in $E^{+}$is called a measure of noncompactness (MNC) on X if $\Phi(\cos \Omega)=\Phi(\Omega)$ for all bounded subsets $\Omega \subset X$, where $c o \Omega$ stands for the closed convex hull of $\Omega$. The MNC $\Phi$ is said:

(1) Monotone if for all bounded subsets $\Omega_{1}, \Omega_{2}$ of $\mathrm{X}$ we have:

$\left(\Omega_{1} \subseteq \Omega_{2}\right) \Rightarrow\left(\Phi\left(\Omega_{1}\right) \leq \Phi\left(\Omega_{2}\right)\right) ;$

(2) Nonsingular if $\Phi(\{\mathrm{a}\} \cup \Omega)=\Phi(\Omega)$ for every a $\epsilon \mathrm{X}$ ,$\subset \subset X$;

(3) Regular if $\Phi(\Omega)=0$ if only if $\Omega$ is relatively compact in $\mathrm{x}$.

One of the most important examples of MNC is the noncompactness measure Of Hausdorff $\beta$ defined on each bounded subset $\Omega$ of X by $\beta(\Omega)=\inf \{\varepsilon>0 ; \Omega$ can be covered by a finite number of balls of radii smaller than $\varepsilon$ \}.for all bounded subset $\Omega, \Omega_{1}, \Omega_{2}$ of X,

$$
\begin{gathered}
\text { (1) } \beta\left(\Omega_{1}+\Omega_{2}\right) \leq \beta\left(\Omega_{1}\right)+\beta\left(\Omega_{2}\right), \text { where } \Omega_{1}+ \\
\Omega_{2}=\left\{\mathrm{x}+\mathrm{y}: \mathrm{x} \in \Omega_{1}, \mathrm{y} \in \Omega_{2}\right\} \\
\text { (2) } \beta\left(\Omega \bigcup_{1} \Omega\right)_{2} \leq \max \left\{\beta(\Omega){ }_{1} \beta(\Omega)\right\}_{;}
\end{gathered}
$$

(3) $\beta(\lambda \Omega) \leq \lambda \beta \Omega$ for any $\lambda \epsilon \mathrm{R}$;

(4) If the map Q: $\mathrm{D}(\mathrm{Q}) \quad \subseteq \mathrm{X} \rightarrow \mathrm{Z}$ is Lipschitz continuous with constants k,then $\beta_{Z}(\mathrm{QZ}) \leq$ $\mathrm{k} \beta(\Omega)$ for any bounded subset $\Omega \subset D(Q)$, where $\mathrm{Z}$ is a Banach space.

Definition 2.2: A two parameter family of bounded linear operators $\mathrm{U}(\mathrm{t}, \mathrm{s}), 0 \leq \mathrm{s} \leq \mathrm{t} \leq \mathrm{b}$ on $\mathrm{X}$ is called an evolution system if the following two conditions are satisfied: $\leq \mathrm{t} \leq \mathrm{b}$

(i) $\mathrm{U}(\mathrm{s}, \mathrm{s})=\mathrm{I}, \mathrm{U}(\mathrm{t}, \mathrm{r}) \mathrm{U}(\mathrm{r}, \mathrm{s})=\mathrm{U}(\mathrm{t}, \mathrm{s})$ for $0 \leq \mathrm{s} \leq \mathrm{r}$

(ii) $\mathrm{U}(\mathrm{t}, \mathrm{s})$ is strongly continuous for $0 \leq \mathrm{s} \leq \mathrm{t} \leq \mathrm{b}$

And there exists $M_{1}>0$ such that $U(t, s) \leq M_{1}$ for any $(\mathrm{t}, \mathrm{s}) \in \mathrm{T}$.

Definition 2.3: A function $\mathrm{x}(\cdot) \in \mathrm{PC}([0, \mathrm{~b}] ; \mathrm{X})$ is a mild solution of(1.7)-(1.9) if

$$
\begin{aligned}
& X t=U t, 0 x_{0}-M x_{t} \\
& +U(t, s) f s, x s \\
& 0 \\
& +\quad(s, \tau, x(\tau)) d \tau+B u(s) d s \\
& +\underset{0<t_{i}<t}{ } U t, t \underset{i}{I}(\underset{i}{x}(t)) \text {, for all } \mathrm{t} \in[0, \mathrm{~b}],
\end{aligned}
$$

where $x 0+M x=x_{0}$.

Definition 2.4: The system (1.7) -(1.9) is said to be controllable on the interval $\mathrm{J}$ if for every initial function $\varphi \in \mathrm{D}$ and $\mathrm{x}_{1} \in \mathrm{X}$, there exists a control $\mathrm{u} \epsilon \mathrm{L}_{2}$ $(\mathrm{J}, \mathrm{V})$ such that themild solution $\mathrm{x}(\cdot)$ of $(1.7)-(1.9)$ satisfies. $x b=x_{1}+M x$.

Definition 2.5: A countableset $\left\{f_{n}\right\}_{n=1}^{+\infty} \subset L^{1}([0, \mathrm{~b}] ; \mathrm{X})$ is said to be semicompact if:

(1) The sequence $\left\{f_{n}\right\}_{n=1}^{+\infty}$ is relatively compact in $\mathrm{X}$ for a.e. $\mathrm{t} \epsilon[0, \mathrm{~b}]$

(2) There is a function $\mu \epsilon L^{1}\left([0, \mathrm{~b}] ; R^{+}\right)$satisfying $\sup _{n \geq 1} f_{n}(t) \leq \mu(t)$ for a.e.

$\mathrm{t} \in[0, \mathrm{~b}]$

Lemma 2.1: Let $\left\{f_{n}\right\}_{n=1}^{+\infty}$ be a sequence of function in $L^{1}\left([0, \mathrm{~b}] ; R^{+}\right)$.Assume that there exist

$\mu, \eta \in L^{1}\left([0, \mathrm{~b}] ; R^{+}\right)$satisfying $\sup _{n \geq 1} f_{n}(t) \leq \mu(t) \quad$ and $\beta\left(f_{n} t \quad{ }_{n=1}^{+\infty}\right) \leq \eta(t)$ a.e. $\mathrm{t} \epsilon_{t}[0, \mathrm{~b}]$. Then for all $\mathrm{t} \epsilon$

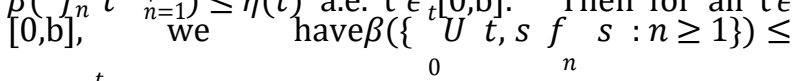
$2 M_{1}{ }_{0} \eta(s) d s$.

Lemma 2.2: Let (Gf) ( $\mathrm{t})={ }_{0}^{t} U t, s f(s) d s$. If $\left\{f_{n}\right\}_{n=1}^{+\infty} \subset L^{1}([0, \mathrm{~b}] ; \mathrm{X})$ is semicompact then the set $\left\{G f_{n}\right\}_{n=1}^{+\infty}$ is relatively compact in $\mathrm{C}([0, \mathrm{~b}] ; \mathrm{X})$ and moreover, if $f_{n} \rightarrow f_{0}$, then for all $\mathrm{t} \epsilon[0, \mathrm{~b}]$, 
$\left(G f_{n}\right) t \rightarrow\left(G f_{0} t\right.$ as $\mathrm{n} \rightarrow+\infty$.

Lemma 2.3: Let $\mathrm{D}$ be a closed convex subset of a Banach space $X$ and $0 \epsilon D$. Assume that $F: D \rightarrow X$ is a continuous map which satisfies Monch's condition, that is, $\mathrm{M} \subseteq$ Discountable, $M \subseteq$ co 00$\} \bigcup F(M) \Rightarrow M$ is compact. Then, there exists $\mathrm{x} \in \mathrm{D}$ with $\mathrm{x}=\mathrm{F}(\mathrm{x})$.

\section{CONTROLLABILITY RESULTS}

We first give the following hypothesis:

(H1) $A(t)$ is a family of linear operators , $A(t)$ : $D(A)$ $\rightarrow \mathrm{x}, \mathrm{D}(\mathrm{A})$ not depending on $\mathrm{t}$ and dense subset of $\mathrm{X}$, generating an equicontinuous evolution system $\{\mathrm{U}(\mathrm{t}, \mathrm{s}):(\mathrm{t}, \mathrm{s}) \in \Delta\}$, i.e.,

$(\mathrm{t}, \mathrm{s}) \rightarrow\{U t, s x: x \in B\}$ is equicontinuous for $\mathrm{t}>0$ and for all bounded subsets $B$.

(H2) The function $\mathrm{f}:[0, \mathrm{~b}] \times X \rightarrow X$ satisfies:

(i) For a.e. $t \in[o, b]$, the function $\mathrm{f}\left(\mathrm{t},{ }^{-}\right): \mathrm{X} \rightarrow \mathrm{X}$ is continuous and for all $\mathrm{x} \in \mathrm{X}$, the function $f($. $, x):[0, \mathrm{~b}] \rightarrow \mathrm{X}$ is measurable;

(ii) There exists a function $m \epsilon L^{1}\left([0, \mathrm{~b}] ; R^{+}\right)$and a nondecreasing continuous function

$$
\Omega: R^{+} \rightarrow R^{+} \text {such } \quad \text { that } f(t, x) \leq
$$
$m t \Omega(x), \mathrm{x} \in X, t \in[0, b]$ and

$$
\lim _{n \rightarrow+\infty} \text { inf } \frac{\Omega(n)}{n}=0 .
$$

(iii) There exists $\mathrm{h} \epsilon L^{1}\left([0, \mathrm{~b}] ; R^{+}\right)$such that , for any bounded subset $\mathrm{D} \subset X$,

$$
\beta f t, x t \leq \quad t \beta x t \text { fora.e. } t \in[0, b],
$$

where $\beta$ is the Hausdorff MNC

(H3)The function $\mathrm{h}:[0, \mathrm{~b}] \times X \rightarrow X$ satisfies:

(i) For each $\mathrm{t}, s \in[0, \mathrm{~b}]$, the function $\mathrm{h}\left(\mathrm{t}, \mathrm{s},{ }^{\cdot}\right): \mathrm{X} \rightarrow \mathrm{X}$ is continuous and for all $\mathrm{x} \in \mathrm{X}$, the

function $\mathrm{h}(\because, x): x):[0, \mathrm{~b}] \rightarrow \mathrm{X}$ is measurable; that

(ii) There exists a function $m \epsilon L^{1}\left([0, \mathrm{~b}] ; R^{+}\right)$such

$$
\begin{aligned}
& \quad(t, s, x(s)) \underset{x}{\leq} t, s \quad x(s), \quad \mathrm{x} \in X, t, s \in[0, b] \\
& \text { and } \lim _{n \rightarrow+\infty} \text { inf } \frac{\mathrm{x}(n)}{n}=0 .
\end{aligned}
$$

(iii) There exists $\zeta \epsilon L^{1}\left([0, \mathrm{~b}] ; R^{+}\right)$such that , for any bounded subset $\mathrm{D} \subset X$,

$$
\beta \quad t, s, x s \leq \zeta t, s \beta x s \text { for a.e } t \epsilon J \text {, }
$$

For convenience let us take $L_{0} \quad=\max _{0}{ }_{0}^{t} m(t, s) d s$ and $\zeta^{*}=\max ^{t} \zeta(t, s) d s$

(H4) $\mathrm{M}: \mathrm{PC}(\mathrm{J}, \mathrm{X}) \rightarrow \mathrm{X}$ is a continuous compact operator such that

$$
\lim _{y P C \rightarrow+\infty} \frac{M(y)}{y \quad P C}=0 ;
$$

(H5) The linear operator $\mathrm{W}: L^{2} J, V \rightarrow X$ is defined byWu $={ }^{b} U b, s B u(s) d s$ such that:

(i) W has an invertible operators $W^{-1}$ which take values in $L^{2} J, V$ ker $W$ and there

exist positive constants $M_{2}, M_{3}$ such that $B \leq M_{2}$ and $W^{-1} \leq M_{3}$;

(ii) there is $K \in L_{W}^{1} J, R^{+}$such that, for any bounded set $\mathrm{Q} \subset X$

$$
\beta W^{-1} Q t \leq K_{W} t \beta(Q)
$$

(H6) Let $I_{i}: X \rightarrow X$, is $i=1, \ldots, s$ be a continuous operator such that:

(i) There are non decreasing functions $I_{i}: R^{+} \rightarrow$ $R^{+}, i=1, \ldots, s$ such that

$$
I_{i}(x) \leq I X_{i} \text { and } \lim \quad \inf \frac{\mathrm{I}_{\mathrm{i}}(n)}{n}=0, \mathrm{i}=1, \ldots, \mathrm{S} .
$$

(ii) There exist constants $K_{i} \geq 0$, such that $\beta I_{i} x\left(t \leq K_{i} \beta(x(t))\right.$. $\mathrm{i}=1, \ldots, \mathrm{s}$.

(H7) The following estimation holds true:

$$
\begin{aligned}
& \begin{array}{l}
\mathrm{L}=\left(M_{1}+2 M^{2} M_{2} K_{W}{ }_{1}^{1}\right) \\
8 M^{2} M_{2} K_{W}{ }^{1} 11_{1}+\zeta^{*} b{ }_{L}^{L}
\end{array} \quad{ }_{i=1} K_{i}+4 M_{1}+ \\
& 1 \quad L \quad 1 \quad L \\
& \text { Where } M_{1}=\sup \{U t, s,(t, s) \epsilon \Delta\}
\end{aligned}
$$

Theorem: Assume that (H1) - (H7) are satisfied, then the impulsive integro differential system

(1.7)-(1.9) is nonlocally controllable on J, provided that

$$
\begin{aligned}
& \frac{1}{n}\left[C_{1}+C_{2} M\left(x_{n}\right)+C_{3} \Omega \mathrm{n}+C_{4} \mathrm{x}_{\mathrm{n}}(\tau)+\right. \\
& \left.\mathrm{C}_{5} \underset{\mathrm{i}=1}{\mathrm{~s}=1} \mathrm{I}_{\mathrm{i}}(\mathrm{n})\right]<1 .
\end{aligned}
$$

Proof : Using hypothesis (H5)(i),for every $x \in P C(J, X)$,define the control

$$
\begin{aligned}
& u_{x} t=W^{-1} x \quad{ }_{1}-M \underset{n}{x-\mathrm{Ub}}, \underset{0}{\mathrm{M}} \underset{0}{x} \\
& \begin{aligned}
& b \\
&-U_{0} b, s f s, x_{n} s \\
&+ s, \tau, x_{n} \tau d \tau d s
\end{aligned} \\
& -U t, t_{i} I_{i} x_{n} t_{i} \\
& 0<t_{i}<t
\end{aligned}
$$

We shall show that, when using this control, the operator, defined by 


$$
\begin{aligned}
& G x t=U(t, 0)\left(x_{0}-M(x)\right) \\
& +\underset{s}{U(t, s) f s, x s} \\
& +{ }_{0}(s, \tau, x(\tau)) d \tau+B u_{x}(s) d s \\
& +\underset{0<t_{i}<t}{U} U t, t_{i} I_{i} x t_{i}
\end{aligned}
$$

has afixed point. This fixed point is then a solution of the system (1.7)-(1.9). Clearly

$x b=x_{1}-M x=G x b$ which implies that the system (1.7)-(1.9) is controllable.

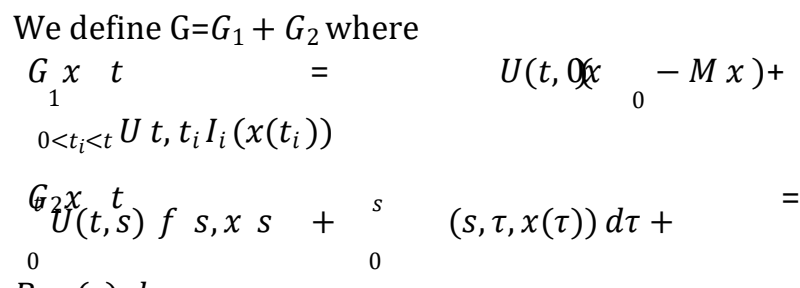

$B u_{x}(s) d s$

for all $t \epsilon 0, b$.subsequently,we will prove that $G$ has a fixed point by using lemma2.3. (Monch fixed point theorem).

Step1:There exist a positive integer $n_{0} \geq 1$ such that $G\left(B_{n_{0}}\right) \subseteq B_{n_{0}}$, where $B_{n_{0}}=\left\{x \in P C J, X: x \leq n_{0}\right\}$.

Suppose the contrary. Then we can find $x_{n} \in P C J, X, y_{n}=G x_{n} \in P C J, X$, such that $x_{n P C} \leq n$

and $y_{n P C}>n$ for every $n \geq 1$.

Now we have

$$
\begin{aligned}
& y_{n} t=\mathrm{U}(\mathrm{t}, 0)\left(\mathrm{x}_{0}-\mathrm{M}\left(x_{n}\right)\right. \\
& \begin{array}{l}
+\underset{0}{U}(t, s) f s, x_{n} s \\
+
\end{array} \\
& +{ }_{0}\left(s, \tau, x_{n}(\tau)\right) d \tau+B u_{x_{n}}(s) \\
& +U t, t_{i} I_{i}\left(x_{n}\left(t_{i}\right)\right) \\
& 0<t_{i}<t \\
& y_{n \quad P C} \leq M_{1} x+M_{0} x \\
& +M_{1} \Omega x_{n P C} \mathrm{~m}_{\mathrm{L}^{1}} \\
& +M_{1} b L_{0} \mathrm{x}_{\mathrm{n}} \tau_{P C} \\
& +M_{1} M_{2}^{\frac{1}{b}} u_{x_{n} L^{2}} \\
& +M \underset{1}{\operatorname{Ix}} \quad i \quad n P C \\
& i=1 \\
& u_{x_{L}} \leq M_{3}\left[x_{1}+M_{1} x_{0}+\left(1+M_{1}\right) M x_{n}\right. \\
& +M_{1} \Omega\left(x_{n P C}\right) m_{L^{1}}
\end{aligned}
$$

$$
\begin{aligned}
& +M_{1} b L_{0} \mathrm{x}_{\mathrm{n}}(\tau) P C \\
& +M_{1} I_{i=1} \quad x_{n} \quad P C
\end{aligned}
$$

Substituting (3.3) in (3.2) we get

1

$<\frac{1}{n} C_{1}+C_{2} M x_{n}+C_{3} \Omega \mathrm{n}+C_{4} \mathrm{x}_{\mathrm{n}} \tau$

$+\mathrm{C}_{5} \quad \mathrm{I}_{\mathrm{i}} \mathrm{n}$

$\mathrm{i}=1$

$w \quad$ ere $C_{1}=M_{1}+{ }_{1} M^{2} M_{2}^{\frac{1}{2}} b^{2} M_{3} \quad x_{0}$

$$
+M_{1} M_{2} \stackrel{\underline{1}}{b^{2}} M_{3} x_{1}
$$

$C_{2}=M_{1}+M_{1} M_{2} b^{2} M_{3}{ }^{1}+M^{2} M_{2} b^{2} M_{3} \frac{1}{-} C_{3}=$ $M_{1} \mathrm{~m}_{\mathrm{L}^{1}}+M_{1} M_{2} b^{2} \bar{M}_{3} \mathrm{~m}_{\mathrm{L}^{1}}$

$$
\mathrm{C}_{4}=M_{1} b L_{0}+M_{1}^{2} M_{2} b^{2}{ }^{3} M_{3} L_{0}, \mathrm{C}_{5}=
$$

$M_{1}+M_{1}^{2} M_{2} b^{\frac{1}{2}} M_{3}$

by passing to the limit as $n \rightarrow+\infty$ in (3.4),we get $1 \leq 0$, which is a contradiction. Thus we deduce that there is $n_{0} \geq 1$ such that $\mathrm{G}\left(B_{n}\right)_{0} \subseteq B_{n}$. 0

Step 2: The operators $G$ is continuous on $P C 0, b ; X$ For thispurpose, we assume that

$x_{n} \rightarrow x \operatorname{in} P C 0, b ; X$.Then by hypothesis (H4) and (H6), we have

$$
\begin{aligned}
& G_{1} x_{n} \rightarrow G_{1} x_{P C} \leq M_{1} M \underset{s}{x_{n}}-M x \\
& +M_{1} I_{i} x_{n} t_{i} \\
& -I_{i} \times t_{i}^{i=1} \\
& \rightarrow G_{2} x \quad{ }_{b} \\
& \leq M_{1} f s, x_{n} s \\
& -f s, x s d s \\
& +M_{1}{ }_{0}^{b}{ }_{0}^{s}\left[\quad s, \tau, x_{n} \tau\right. \\
& -\quad(s, \tau, x(\tau))] d \tau \quad d s \\
& +M_{12} \operatorname{Mr}^{\frac{1}{u}} \\
& -u_{x} L^{2} \\
& u_{x_{n}}-u_{x_{L}} \leq M_{3}\left[M x_{n}-M x\right. \\
& +M_{1} M x_{n}-M x \\
& b \\
& +M_{1} f s, x_{n} s-f s, x s d s
\end{aligned}
$$




$$
\begin{aligned}
& \begin{array}{llll} 
& b & s \\
+M_{1} & & & \\
& 0 & 0
\end{array} \quad s, \tau, x_{n} \tau \\
& -\quad(s, \tau, x(\tau))] d \tau \\
& \underset{1}{+} \underset{i=1}{M} \underset{i}{x}{ }_{n} t_{i}
\end{aligned}
$$$$
-I_{i} \times t_{i}
$$

By domination convergence theorem, we have

$$
\begin{aligned}
G x_{n} & \rightarrow G x_{P C} \leq G_{1} x_{n} \rightarrow G_{1} x_{P C}+G_{2} x_{n} \rightarrow \\
G_{2} x_{C} & \rightarrow 0 \text {, as } \mathrm{n} \rightarrow+\infty \text {, ie., } \mathrm{G} \text { is continuous. }
\end{aligned}
$$

Step 3: G(D) is equicontinuous on every $J_{i}, \mathrm{i}=1$,...s.ie., $\mathrm{D} \subseteq c o \quad 0 \cup G D$ is also equicontinuous on every $J_{i}$. To this end, let $\mathrm{y} \epsilon G(\mathrm{D})$ and $t_{1}, t_{2} \in J_{i}, t_{1} \leq t_{2}$. There is $x \in D$ such that

$$
\begin{aligned}
& y t_{2}-y t_{1} \leq \| U t_{2}, 0-U t_{1}, 0 x_{0}- \\
& M x \| \\
& +\|_{0}^{t_{1}} U t_{2}, s \\
& -U t_{1}, s f s, x s \\
& {\stackrel{\|}{0} d_{0}}^{s} \quad(s, \tau, x(\tau)) d \tau+B u_{x}(s) \\
& +\underset{t_{1}}{t_{2}} t_{2}, 0 f s, x s \\
& +{ }_{0}^{s} s, \tau, x \tau d \tau \\
& +B u_{x} s \quad d s
\end{aligned}
$$

By the equicontinuity property of $U$; s and the absolute continuity of the lebesgue integral, right hand side of the inequality equation (3.8) tends to zero independent of y as $t_{2} \rightarrow t_{1}$.

Therefore G (D) is equicontinuous on every $J_{i}$

Step 4: Assume that $\mathrm{D}=\left\{x_{n}\right\}_{n=1}^{+\infty}$. Since G maps D into an equicontinuous family, $G$ (D) is equicontinuous on $J_{i}$.Hence $\mathrm{D} \subseteq$ co $0 \cup G D$ is also equicontinuous on every $J_{i}$.

Now we shall show that (GD) (t) is relatively compact in $\mathrm{X}$ for each $\mathrm{t} \in J$.

From the compactness of M $(\cdot)$, we have

$$
\begin{aligned}
& \left.\beta \underset{s}{\left(G_{1} x_{n}\right.}(t)\right\}_{n=1}^{\infty} \\
& \leq M_{1} K_{i=1} \beta x t_{i}
\end{aligned}
$$

for $t \epsilon 0, b$.by lemma(2.1), we have

$$
\begin{aligned}
& \beta_{V}\left(\begin{array}{lll}
u_{x_{n}} & s & \infty
\end{array}\right) \\
& \leq K_{W}(s) 2 M_{1}{ }_{0}^{b} s \beta x s d s+2 M_{1} \zeta^{*} b \beta \times s \\
& +M_{i=1}{ }^{s} K_{i} \beta \times t_{i}
\end{aligned}
$$

Then this implies that

$\beta\left(G_{2} x_{n}(t)\right\}^{\infty}{ }_{n=1}$

$$
\begin{aligned}
& \leq 2 M_{1}^{b} s \beta x s d s \\
& +4 M_{1}^{2} M_{2} K_{W}^{b} s d s\left(\quad \begin{array}{c}
b \\
0
\end{array} x s d s\right) \\
& +2 M_{1} \zeta^{*} b \beta x s \\
& +4 M_{1}^{2} M_{2} K_{W} s d s \zeta^{*} b \beta x s \\
& +2 M_{1}^{2} M_{2}{ }_{0}^{b} K_{W} \eta d \eta{ }_{i=1}^{s} K_{i} \beta x t_{i}
\end{aligned}
$$

There fore

$\beta((\mathrm{GD})(\mathrm{t}))$

$$
\begin{aligned}
& \leq M_{1} K_{i} \beta x\left(t_{i}\right) \\
& i=1 \\
& +2 M_{1} \\
& +\underset{1}{4 M_{2}^{2}} M_{0} \underset{W}{s} \underset{W}{d s} \quad s \beta x \stackrel{b}{s} d s
\end{aligned}
$$

$+2 M_{1}$

$$
\begin{gathered}
{ }^{b}{ }^{2} M_{2} M_{W} s d s \zeta^{*} b \beta x s \\
+2 M_{1}^{2} M_{2} K_{W}^{b} \eta d \eta K_{i} \beta x t_{i=1}^{s}
\end{gathered}
$$

we have

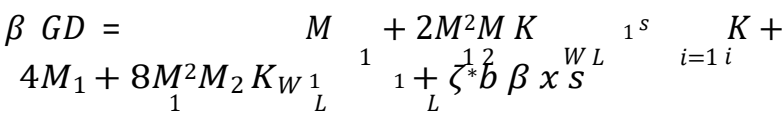

$$
\begin{aligned}
& =L \beta \times S
\end{aligned}
$$

Where $L$ is defined in (H7). Thus, from the Monch's condition, we get

$\beta(D) \leq \beta($ co $0 \cup G D=\beta(G(D)) \leq L \beta(D)$

Which implies that $\beta(D)=0$, since hypothesis (H7) holds. So we have that $\mathrm{D}$ is relatively compact. Finally, due to lemma, $G$ has at least a fixed point and 
thus the system (1.7)-(1.9) is non locally controllable on $[0, \mathrm{~b}]$.

\section{REFERENCES}

Bainov, D.D. and P.S. Simeonov, (1993). Impulsive Differential Equations: Periodic Solutions and Applications, Longman Scentific and Technical Group, England.

Cuevas, C., E. Hernandez and M. Rabello, (2009). The existence of solutions for impulsiveneutral functional differrential equations, Computers and Mathematics with Applications, 58: 744757.

Fan, Z. and G. Li, (2010). Existence results for semilinear differential equations with nonlocal and impulsive conditions. J. Funct. Anal. 258: 1709-1727.
Ji, S., G. Li and M. Wang, (2011). Controllability of impulsive differential systems with nonlocal conditions. Appl. Math. Comp. 217: 6981-6989.

Chen, L. and G. Li, (2010). Approximate controllability of impulsive differential equations with nonlocal conditions. Int. J. Nonlinear Sci. 10: 438-446.

Guo, M., X. Xue and R. Li, (2004).Controllability of impulsive evolution inclusions with nonlocal conditions. J. Optim. Theory Appl. 120: 355-374.

Jose A.M., C. Ravichandran, R. Margarita and J.J. Trujillo, (2013). Controllability results for impulsive mixed-type functional integrodifferential evolution equations with nonlocal conditions.

Anguraj, A. and M. Mallika Arjunan, (2009). Existence results for an impulsive neutral integrodifferential equations in Banach spaces. Nonlinear Studies. 16(1): 33-48. 